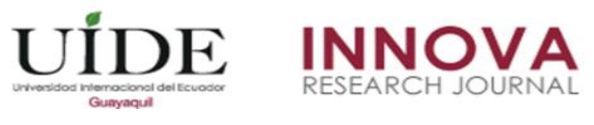

INNOVA Research Journal, ISSN 2477-9024

(Julio, 2018). Vol. 3, No.7 pp. 95-113

DOI: https://doi.org/10.33890/innova.v3.n7.2018.603

URL: http://revistas.uide.edu.ec/index.php/innova/index

Correo: innova@uide.edu.ec

\title{
La propiedad industrial en Venezuela: desarrollo institucional y experiencia normativa (1955-2017)
}

\section{The industrial property in Venezuela: institutional development and normative experience (1955-2017)}

Carolina Lourdes Rodríguez Aguilera

Aura Esther Troconis Troconis

Universidad Simón Bolívar, Venezuela

Autor para correspondencia: rodirguezcarol@usb.ve, atroconis@usb.ve

Fecha de recepción: 20 de enero de 2018 - Fecha de aceptación: 25 de julio de 2018

Resumen: Los activos intangibles representan para los países retos y oportunidades que se traducen en creaciones a favor de su desarrollo económico y social. El resguardo de este patrimonio es responsabilidad de los Estados junto a los actores que integran los Sistemas Nacionales de Ciencia, Tecnología e Innovación (SNCTI), mediante la generación de políticas públicas y legislaciones cónsonas con las expectativas nacionales, el comercio y las relaciones internacionales.

El objetivo de este artículo es presentar un recorrido institucional y normativo del desarrollo de la propiedad industrial en Venezuela, mediante la visión de los entes, las legislaciones y políticas públicas desarrolladas en el país desde 1955 al 2017.

La metodología utilizada en la investigación es fundamentalmente teórica y de carácter documental. En ese sentido, esta pesquisa es descriptiva y analítica, por consiguiente, se estructuró partiendo de la revisión de diversas fuentes nacionales e internacionales de organizaciones especializadas, bibliográficas y hemerográficas, entre las que se encuentran, informes, actas, leyes, acuerdos, decisiones, proyectos y planes generados que reflejan tanto los antecedentes como la situación actual de dichos procesos.

Palabras Claves: propiedad industrial; Venezuela; normativa; políticas públicas

\begin{abstract}
Intangible assets represent challenges and opportunities for the countries, that are translated into creations in favor of their economic and social development. The protection of this heritage is the responsibility of the States along with the actors that make up the National Science, Technology and Innovation Systems (Sistemas Nacionales de Ciencia, Tecnología e Innovación - SNCTI), through the generation of public policies and legislations in harmony with national expectations, trade and international relations.

The objective of this article is to present an institutional and normative journey of the development of industrial property in Venezuela, through the vision of the entities, the legislations and public policies developed in the country from 1955 to 2017.

The methodology used in the research is mainly theoretical and documentary. In that sense, this research is descriptive and analytical, therefore was structured on the basis of the revision of various national and international sources of specialized organizations, bibliographical and newspaper, among which are, reports, minutes, laws, agreements, decisions, projects and plans generated that reflect both the background and the current situation of such processes.
\end{abstract}

Key words: industrial property; Venezuela; regulation; public policies 


\section{Introducción}

Tradicionalmente la propiedad intelectual se divide en dos grandes ramas, a saber: el Derecho de Autor y la Propiedad Industrial, objeto de este artículo; no obstante, en el seno de la Comunidad Andina (CAN) se generaron nuevas formas de protección, como la referida a la obtención de nuevas variedades vegetales contenida en la Decisión 345 de 1993. Igualmente, y considerando el valor que han adquirido los aspectos asociados a la biodiversidad y conservación de las especies, en el año 1996, se creó en el marco del mismo organismo andino, la Decisión 391, en la que se expresan los derechos de acceso a los recursos genéticos y de protección de los conocimientos tradicionales y las expresiones culturales tradicionales.

Por su parte, en el espacio multilateral, estos derechos se amplían en octubre del año 2000, gracias a la labor del Comité Intergubernamental de la Organización Mundial sobre Propiedad Intelectual y Recursos Genéticos, Conocimientos Tradicionales y Folclore (CIG), que busca generar un instrumento jurídico de orden internacional que garantice la protección de los aspectos que dan origen a la labor de dicho Comité.

El tema de la propiedad industrial es relevante para el desarrollo de los países, y comprende las creaciones de la mente, por lo que está estrechamente relacionado a los activos intangibles, al conocimiento materializado en las invenciones, las obras literarias y artísticas, los símbolos, nombres, imágenes, dibujos y modelos, aspectos que desempeñan un papel importante en la promoción de la innovación, la creación de empleo, el acceso a los medicamentos, la creatividad cultural, la diversidad y protección ancestral. Asimismo, involucra la relación entre los diversos actores de los Sistemas de Ciencia, Tecnología e Innovación (SCTI), la transferencia de tecnología, la inversión extranjera, la promoción de exportaciones y el comercio.

Todos estos elementos encuentran en las leyes y normas el espacio mediante el cual, el conocimiento no solo encuentra un mecanismo de protección legal para los derechos y beneficios económicos que pudiesen generarse, sino también, el reconocimiento y la reivindicación de los derechos morales de los individuos, empresas, institutos de investigación, universidades, laboratorios, creadores de ese conocimiento.

El alcance de la propiedad industrial y las políticas públicas vinculadas a ésta, son hoy en día un componente esencial de los planes nacionales de desarrollo y los planes de ciencia, tecnología e innovación, lo que genera una mayor demanda en cuanto a la forma de adoptar, aplicar y adaptar el marco institucional y jurídico de cada país, para responder a las necesidades y expectativas que en torno a la propiedad industrial se generan internacionalmente.

Los derechos contemplados en materia de propiedad industrial forman parte de la denominada economía del conocimiento, que hoy cuenta en el mundo de las relaciones internacionales con una nueva jerarquía, constituyendo lo que podría denominarse las fronteras invisibles, puesto que tocan relaciones que van mucho más allá de la compra- venta o comercialización de un producto, servicio o nueva tecnología, por lo que estamos en presencia del surgimiento de:

...una nueva literatura en teoría del management, en economía de la innovación, en competitividad, en la ciencia política que pone cada vez más 
énfasis en el papel de las instituciones, las relaciones sociales, las normas y valores culturales, así como en los mecanismos de coordinación entre los agentes con intereses diversos como elementos centrales para desarrollar estrategias competitivas..." (Zurbriggen y González, 2010:15).

En el caso de Venezuela luego de salir de la Comunidad Andina (CAN) en el 2006, la situación particular que vive el país en lo que respecta a la propiedad industrial, lo colocó en un escenario de retroceso con una Ley de Propiedad Industrial generada en 1955 contenida en la Gaceta Oficial $N^{\circ} 25.227$, con lo cual pasa a ser uno de los países de América Latina con la legislación más desactualizada, en comparación con otros países del continente como: Argentina (2007), Brasil (2015), Ecuador (2016), Chile (2012), Colombia (2013), México (2016), Perú (2009), Uruguay (2004) y Paraguay (2005) (OMPI http://www.wipo.int/wipolex/es/results.jsp?countries=\&cat_id=12).

Sin embargo, un aspecto fundamental tiene que ver con que no basta con solo tener leyes, acuerdos, convenios, tratados, sino hacerlos cumplir y que tengan efecto en las sociedad y sus beneficiarios particulares (naturales o jurídicos), debido a que la protección de los derechos de propiedad industrial pueden contribuir en gran medida a fomentar las inversiones, disminuir el riesgo país, acercar a nuevos socios, fomentar las negociaciones de transferencia de tecnología, la creación "clusters" o "spin off" asociadas con universidades, parques tecnológicos, así como, la promoción del empleo y el incremento por esta vía de los ingresos fiscales.

La violación de estos derechos, trae como consecuencia que se asfixie a la creatividad, estancándose la innovación, lo que se traduce en una disminución de la inversión nacional en la materia, y con esto, la creación de un cerco que limita las actividades de investigación y desarrollo, por tanto, se requiere un sistema judicial consistente para hacer frente a los delitos y controversias que se generen y que contribuyan y apoyen la vinculación entre los países en este sentido.

Todo lo antes expuesto, destaca la importancia de estos aspectos a nivel nacional e internacional, por lo que, en este artículo se analizará la propiedad industrial en Venezuela, desde la perspectiva de su desarrollo institucional y experiencia normativa, mediante el abordaje de la historia y evolución de la misma a través de los entes, legislaciones y políticas públicas desarrolladas en el país desde 1955 al año 2017.

\section{La propiedad industrial en Venezuela}

\section{Desarrollo institucional y experiencia normativa}

En este apartado se realizará un acercamiento general a los entes, legislaciones y políticas públicas desarrolladas en Venezuela en materia de propiedad industrial. Cabe destacar que en 1955, la propiedad industrial en Venezuela se regía por el Departamento de Propiedad Industrial

y Comercial del Ministerio de Fomento, y con la aprobación de la Ley de Propiedad Industrial en 1956, publicada en la Gaceta Oficial № 25.227 de fecha 10 de diciembre de 1956, no sólo se generó una legislación en la que se protegieran y desarrollaran al unísono todos los aspectos sobre la materia, sino también, surgió la necesidad de crear un ente especializado que se 
encargase tanto administrativamente como legalmente de todos los aspectos sobre la propiedad industrial en Venezuela.

Es importante señalar que la Ley de Propiedad Industrial de 1956, establece las disposiciones en materia de Patentes, los Modelos y Dibujos Industriales, las Marcas Comerciales, del Registro de la Propiedad Industrial, de los Derechos de Registro, Anualidades de Patentes e Impuesto a la Solicitudes, de los Agentes de la Propiedad Industrial, del Boletín y de la Publicaciones, Procedimientos, de las Marcas, de las Cesiones y Cambios de Nombres, las Penalizaciones, Clasificación y Disposiciones transitorias.

Dentro de este contexto, a partir de la Ley de Propiedad Industrial de 1956, se creó en ese mismo año, el Registro de Propiedad Industrial adscrito al Ministerio de Fomento. Según (Salazar, 2010: 149), "los años venideros, es decir, desde 1956 a 1989, se caracterizaron por un total oscurantismo en esta disciplina". Por otra parte, a partir de 1989, con el segundo gobierno del ex presidente Carlos Andrés Pérez, resurgió en Venezuela el interés sobre el tema de la propiedad intelectual, motivando la creación en noviembre de 1992, del Servicio Autónomo Registro de la Propiedad Intelectual (SARPI) adscrito al Ministerio de Fomento, otorgándosele una nueva y mayor autonomía financiera.

Es pertinente indicar que, en 1997, se realizó una reforma de la Administración Pública Central, y la propiedad intelectual y el derecho de autor pasaron a ser competencia del Ministerio de Comercio, anterior Ministerio de Fomento. Solo hasta ese año, el SARPI y la Dirección Nacional de Derecho de Autor (DNDA) se integraron bajo la figura del Servicio Autónomo de Propiedad Intelectual (SAPI), creado mediante el Decreto $\mathrm{N}^{\circ} 1.768$ de fecha 25 de marzo de 1997, publicado en la Gaceta Oficial $N^{\circ} 36.192$ de fecha 24-04-97, tiempo desde el cual, este ha sido el único organismo encargado de esta competencia en el país, cambiando en algunos casos de ministerio de adscripción, sin dejar de ejercer sus funciones en la materia.

Dicho ente fue un importante actor a considerar dentro del marco regulatorio y condiciones que rigieron la adhesión de Venezuela al MERCOSUR, desde que comenzó el proceso en el año 2005 con la firma del Acuerdo Marco para la adhesión de Venezuela al MERCOSUR.

Hoy en día, los entes del Estado encargados de dictar las pautas y políticas públicas en materia de comercio, propiedad intelectual y ciencia y tecnología son el Ministerio del Poder Popular para el Comercio y el Ministerio del Poder Popular para la Ciencia, Tecnología e Innovación, actualmente denominado Ministerio del Poder Popular para Educación Universitaria, Ciencia y Tecnología, instituido mediante Gaceta $\mathrm{N}^{\circ} 40.489$ del 3 de septiembre de 2014, Decreto $N^{\circ} 1.226$, con competencia en el ámbito de las políticas públicas del Ejecutivo Nacional, en cuanto a la Educación Universitaria, Ciencia y Tecnología.

Específicamente, en lo que respecta al Ministerio del Poder Popular para el Comercio, su principal competencia radica en:

... las políticas públicas de comercio nacional e internacional; el control antimonopolio, de carteles y otras prácticas similares; la producción y oferta 
nacional en la provisión de bienes, obras y servicios; la inversión extranjera; la propiedad intelectual; el acceso de las personas a bienes y servicios; la calidad, normalización, certificación; la acreditación, metrología y reglamentos técnicos para producción de bienes y servicios, y en materia de arrendamiento inmobiliario con fines comerciales"(http://www.mincomercio.gob.ve, 2018).

Este organismo delega las funciones en materia de propiedad intelectual, en el Servicio Autónomo de Propiedad Intelectual (SAPI), que ejerce desde hace 18 años, la competencia que le corresponde al Estado, en este sentido.

Es preciso señalar que, el SAPI mantiene la misma estructura organizativa desde su creación, conformada por una Dirección General, la Dirección de Registro de la Propiedad Industrial, Dirección Nacional de Derecho de Autor, Dirección de Asesoría Jurídica, Dirección de Soporte Administrativo y Dirección de Difusión y Cooperación, que orientan sus actividades hacia:

... la administración de la concesión de derechos a los inventores sobre sus creaciones, a través de las patentes de invención, mejoras, dibujos y diseños industriales; a los comerciantes o personas naturales sobre los signos que utilizan para distinguir sus productos y servicios en el mercado, mediante las marcas, denominaciones comerciales y lemas comerciales; el registro, fiscalización e inspección sobre los derechos de autor y los derechos conexos, en el ámbito administrativo. (http://www.sapi.gob.ve/?page_id=32, 2018).

Ahora bien, entre los diferentes objetivos del SAPI relacionados con la defensa, protección, promoción, incentivo, cooperación y coordinación de las actividades sobre la propiedad intelectual, es importante resaltar dos de ellos, estos son:

a) Cooperar y negociar en el ámbito local, regional y mundial, con los Estados, las organizaciones y los pueblos, para la protección y desarrollo de la propiedad intelectual de acuerdo con los principios fundamentales de la Constitución de República Bolivariana de Venezuela.

b) Coordinar la administración de los convenios nacionales e internacionales para la consolidación del Sistema Nacional de la Propiedad Intelectual y suscripción de nuevos compromisos..." (http://www.sapi.gob.ve/?page_id=32, 2018).

En ambos casos, puede observarse la relevancia que implica la vinculación con otros Estados, organizaciones y convenios de tipo nacional e internacional.

Es interesante observar que, posterior a la denuncia de Venezuela de la CAN, el 22 de abril de 2006, el SAPI expidió una comunicación oficial el 12 de septiembre de 2008, mediante la cual, le informaba al público en general, que como consecuencia de la decisión tomada por el Estado, se restituiría la aplicación en su totalidad de la Ley de Propiedad Industrial de 1956, como la ley vigente en el país, cuya aplicación se realizaría íntegramente, siempre y cuando no contradiga ningún aspecto contentivo en la Constitución nacional vigente. Además, se precisó 
que, junto a esta Ley, el ordenamiento jurídico nacional en la materia se regiría por la Ley Orgánica de Procedimientos Administrativos (1981) y la Ley Orgánica de la Administración Pública (2014).

En consecuencia, algunos de los aspectos que pueden destacarse como resaltantes al momento de retomar la Ley de Propiedad Industrial de 1956, tienen que ver por ejemplo, con la reducción en el campo de patentabilidad en el sector de las medicinas y alimentos; otro elemento es, que se vuelve a la definición por separado de dibujo industrial y modelo industrial que se agrupaban en la Decisión Andina 486 como diseño industrial; por otro lado, se reduce el tiempo por el derecho de uso exclusivo de una marca de 15 años a 10 años; además, se pierde la posibilidad de que la autoridad administrativa dicte medidas cautelares, ya que el SAPI no tiene esa función.

Asimismo, en cuanto a los sistemas y circuitos integrados, la Ley de Propiedad Industrial de 1956, no prevé ningún articulado al respecto, con lo cual queda sin efecto, protección alguna en este sentido. Por consiguiente, se advierte la necesidad de dictar una legislación que se ajuste a la realidad tecnológica internacional, ya que en la actualidad Venezuela está dejando sin protección legal múltiples aspectos en materia de propiedad industrial.

Es necesario enfatizar que el SAPI tiene como fundamento institucional, la Constitución de la República Bolivariana de Venezuela, y las líneas estratégicas del Plan de Desarrollo Económico y Social de la Nación, establecidas en el Proyecto Nacional Simón Bolívar 20072013; así como, la Ley de Propiedad Industrial de 1956.

Con referencia a la Constitución de Venezuela de 1999, la propiedad intelectual está contemplada en el Capítulo VI sobre los Derechos Culturales y Educativos, específicamente en el artículo 98, que dispone que el Estado protegerá la propiedad intelectual; y el Capítulo VIII de los Derechos de los Pueblos Indígenas, artículo 124, en el cual se garantizan los derechos de propiedad intelectual colectiva de los conocimientos, tecnologías e innovaciones de los pueblos indígenas; y el Capítulo II, sobre la Competencia del Poder Público Nacional, en particular, el apartado 32, en el cual se fija posición sobre el ejercicio del Estado sobre la de propiedad intelectual, artística e industrial; la del patrimonio cultural y arqueológico nacional.

En cuanto al Proyecto Nacional Simón Bolívar 2007-2013 (2007), es pertinente advertir que en el apartado IV-3. Estrategias y Políticas dentro de los sub puntos IV-3.13 y IV-3.13.1 sobre mejorar el apoyo institucional para la ciencia, la tecnología y la innovación, se hace referencia a "Simplificar los trámites para la obtención de patentes y reducir costos", y en la sección IV-3.13.5 se propone "Actualizar el banco de patentes y modernizar los sistemas de información" (p. 28).

Cabe agregar que los lineamientos del Plan de la Patria 2013-2019 (2013), en cuanto a lo que se refiere al Objetivo Estratégico 3 se destaca la necesidad de convertir a Venezuela en un país potencia en lo social, económico y lo político, dentro de la gran potencia naciente de América Latina y el Caribe, que garanticen la conformación de una zona de paz en Nuestra América. En ese orden de ideas, el sub objetivo 3.2.1.11. Del Plan indicado se insta a "Impulsar mecanismos y prácticas de vigilancia tecnológica para la protección de marcas y patentes 
venezolanas en el extranjero, incluyendo el monitoreo y las acciones defensivas necesarias para la salvaguarda de este patrimonio intangible de los venezolanos y las venezolanas" (p. 19).

Por su parte, el Ministerio del Poder Popular para la Educación Universitaria, Ciencia y Tecnología (MPPEUCyT), tiene entre sus competencias la formulación de “...las políticas en materia de Ciencia, Tecnología, Innovación y sus aplicaciones; el Sistema Nacional de Ciencia, Tecnología e Innovación, las telecomunicaciones, las tecnologías de información y su desarrollo..." (http://www.mppeuct.gob.ve/, 2018).

En consecuencia, la misión del MPPEUCyT involucra entre otros aspectos, la consolidación de las capacidades científico-tecnológicas, y "la definición de un modelo científico-tecnológico propio que oriente y rija el Sistema Nacional de Ciencia, Tecnología e Innovación (SNCTI)" (http://www.mppeuct.gob.ve, 2018) con lo cual, es evidente su injerencia y protagonismo en los procesos inherentes a la propiedad intelectual.

Entre las políticas de ese organismo nacional es necesario rescatar dos (2), donde la propiedad intelectual juega un importante papel; estas son las siguientes:

el estímulo a los proyectos de investigación y desarrollo tecnológico orientados a la generación de conocimientos, la transferencia tecnológica y la innovación en áreas estratégicas; y el impulso a los procesos de aplicación de los conocimientos y tecnologías en el desarrollo y fortalecimiento del sistema productivo regional y nacional. (http://www.mppeuct.gob.ve/, 2018).

Adicionalmente, según el Plan Nacional de Ciencia, Tecnología e Innovación 2005 2030, elaborado por la Dirección General de Planificación Prospectiva en el año 2005, las normas internacionales de propiedad intelectual, según las normas de la Organización Mundial del Comercio (OMC), representarían un riesgo, por lo que, se propuso "establecer políticas de protección a la industria farmacéutica" (http://www.mppeuct.gob.ve/ 2006: 45).

Ese lineamiento dificulta el desarrollo de un sector que a nivel nacional cuenta con instituciones, empresas y profesionales capaces de generar productos de calidad en beneficio de la población venezolana. El país importa gran parte de los medicamentos consumidos por su población, con lo cual se encarece su adquisición, por tanto, generar políticas públicas que estimulen su producción y protección significaría una disminución en cuanto a los costos y un mayor acceso a los mismos.

Igualmente, en el Plan Nacional de Ciencia, Tecnología e Innovación 2005 -2030, elaborado por el Ministerio de Ciencia y Tecnología (MCT), se planteó como estrategia, el "apoyo científico-tecnológico dirigido al resguardo de la propiedad intelectual colectiva de los saberes estratégicos de los pueblos originarios indígenas y los pueblos afrodescendientes venezolanos y latinoamericanos" (MCT, 2006: 87). Salvo estos dos apartados, no existe otra mención a los aspectos relativos a la propiedad intelectual, en ninguna de sus acepciones ni tipos de protección. 
Ahora bien, siguiendo las normativas desarrolladas por el Estado Venezolano, como parte de las políticas públicas relacionadas con la propiedad industrial, debe hacerse referencia a la Ley Orgánica de Ciencia Tecnología e Innovación (LOCTI) del año 2010 y el Reglamento Parcial de la LOCTI (2011) sobre los aportes, el financiamiento, los resultados y la ética en la investigación, tecnología e innovación. En especial, porque los artículos 19, 20 y 21 de la LOCTI confirman la autoridad del SAPI como ente rector, coordinador de las políticas de propiedad intelectual, así como, su obligación para apoyar promocionar y difundir las invenciones y las innovaciones populares en beneficio para la población. Del mismo modo, el artículo 27 de la LOCTI plasma la prioridad que otorga el Estado a las actividades que se detallan a continuación:

proyectos de innovación relacionados con actividades que involucren la obtención de nuevos conocimientos o tecnologías en el país, con participación nacional en los derechos de propiedad intelectual, en las áreas prioritarias establecidas por la autoridad nacional con competencia en materia de ciencia, tecnología, innovación y sus aplicaciones (LOCTI, 2010: 25).

Mientras que, a través del artículo 26 del Reglamento LOCTI (2011), se establece que todos los aspectos susceptibles de financiamiento, estén o no protegidos por derechos de propiedad intelectual, se canalizarán por medio del Fondo Nacional de Ciencia Tecnología e Innovación (FONACIT), sin hacer mención a ningún otro elemento sobre la materia.

Conviene precisar, que entre los entes adscritos al MPPEUCyT, está la Fundación Observatorio Nacional de Ciencia, Tecnología e Innovación (ONCTI), que incluye los temas de propiedad intelectual en el Reglamento del Programa de Estímulo a la Innovación e Investigación (PEII), parcialmente modificado en Reunión Extraordinaria $N^{\circ}$ 04, del 11 de enero del año 2011. De este modo, el Párrafo Primero del Capítulo II de los Incentivos de dicho Reglamento, indica que los investigadores pueden optar a múltiples programas de financiamiento, entre los que se encuentran "...gestiones para el registro de Derecho de Propiedad Intelectual...” (ONCTI, 2011: 9).

Así también, en el marco legal venezolano, otro conjunto de leyes que resguardan entre sus contenidos a la propiedad intelectual, desde diferentes enfoques y objetivos, son las siguientes:

a) La Ley Orgánica del Trabajo, los Trabajadores y las Trabajadoras de 2012, publicada en la Gaceta Oficial $\mathrm{N}^{\circ}$ 6.076: en el Capítulo IV de las Invenciones, Innovaciones y Mejoras, particularmente, los artículos que van del 320 al 329 desarrollan las normativas para el trabajador sobre la fuente del conocimiento científico, humanístico y tecnológico; la normativa aplicable; la clasificación de las invenciones, innovaciones o mejoras realizadas por los trabajadores; lo que se considera como invenciones, innovaciones o mejoras; las condiciones de las innovaciones libres u ocasionales; lo que se reconoce como invenciones, innovaciones y mejoras en el sector público y privado; la propiedad 
de las invenciones libres u ocasionales; los derechos morales de los inventores; y los derechos del trabajador o de la trabajadora no dependiente.

Un elemento de suma relevancia presente en estos artículos, es la propiedad de las invenciones, innovaciones o mejoras, sobre todo, en el ámbito público, debido a que se establece que se considerarán del dominio público, si estas provienen o son financiadas por presupuesto público. Esta disposición normativa difiere de la propia Ley de Propiedad Industrial (1956) que en su artículo 3 preserva el derecho de propiedad del particular cuando indica que "se presume que es propietario de un invento, mejora o modelo o dibujo industrial, o de una marca, lema o denominación comercial, o introductor de un invento o mejora, la persona a cuyo favor se haya hecho el correspondiente registro" (p. 1).

Por su parte, en el caso del sector privado, estos derechos se mantendrán mientras exista la relación de trabajo con la empresa, fijándose equitativamente la distribución de las ganancias previa aprobación del inspector del trabajo.

b) Ley Orgánica del Sistema Económico Comunal publicada en la Gaceta Oficial № 6.011, Extraordinaria, del 21 de diciembre de 2010: en la Sección V de la Autoría Intelectual en la Producción Comunal, su Registro y Aprovechamiento, reconocen el conocimiento generado desde la Práctica Productiva del Trabajo; la Propiedad Social y Autoría Intelectual y los Incentivos al Conocimiento, desde la visión del trabajo comunitario.

c) Ley Orgánica de Pueblos y Comunidades Indígenas prevista en la Gaceta Oficial $\mathrm{N}^{\circ}$ 38.344, del 27 de Diciembre de 2005: en el Título IV de la Educación y la Cultura, y Capítulo V de los Conocimientos y la Propiedad Intelectual Colectiva de los Pueblos Indígenas, se presentan las formas sobre el derecho a la propiedad colectiva de los pueblos y comunidades indígenas; del uso de los recursos genéticos; de la protección y defensa de la propiedad intelectual indígena y de las acciones legales contenidas en los artículos 101 al 104.

d) La Ley Orgánica de Aduanas publicada en la Gaceta Oficial $\mathrm{N}^{\circ} 5.353$ Extraordinario, del 17 de junio de 1999: que en el Titulo IV sobre Medidas en Aduanas sobre Propiedad Intelectual, se refiere en los artículos 87 y 88, a la custodia del ingreso al país de mercancías o mercaderías que sean ilegales y violen los derechos de propiedad intelectual, así como, la constante información al ente competente en la materia (SAPI), sobre cualquier irregularidad al respecto. Esta Ley fue reformada parcialmente mediante Ley publicada en Gaceta Oficial de la República Bolivariana de Venezuela (Gaceta Oficial) $\mathrm{N}^{\circ} 38.875$, de fecha 21 de febrero de 2008, y luego reformada parcialmente mediante el Decreto $N^{\circ} 1416$ publicado en la Gaceta Oficial No 6155 Extraordinario, el 19 de noviembre de 2014, pero se mantienen los aspectos mencionados anteriormente.

e) Ley de Diversidad Biológica (LDB) publicada en la Gaceta Oficial Extraordinaria $\mathrm{N}^{\circ}$ 5.468, del 24 de mayo de 2000: en esta ley se expresa categóricamente, la situación sobre las patentes y otras formas de propiedad intelectual, en los Capítulos II y III de la Protección y Reconocimiento de los Conocimientos Tradicionales de los Pueblos y Comunidades Indígenas y Locales, y en el Capítulo IV del Desarrollo y de la Transferencia de Biotecnología, todos hacen hincapié sobre la propiedad colectiva de los 
pueblos y comunidades indígenas, con el objeto de proteger el conocimiento ancestral, las costumbres y tradiciones de los pueblos indígenas "relativas a las creaciones o descubrimientos de productos y procedimientos en materia de biotecnología" (LDB, 2000:14).

Otros entes, organismos, disposiciones que trabajan directa o indirectamente el tema de la propiedad intelectual en Venezuela son: el Comando Anti-Piratería (COMANPI); el Grupo de Acción Nacional Antipiratería (GANA); el Instituto para la Defensa de las Personas en el Acceso a los Bienes y Servicios (INDEPABIS), actualmente Superintendencia de Precios Justos; la Superintendencia para la Promoción y Protección de la Libre Competencia (PROCOMPETENCIA); y la Superintendencia de Inversiones Extranjeras (SIEX) del Ministerio de Poder Popular para el Comercio.

Del mismo modo, es importante resaltar que durante el año 2017 el Servicio Autónomo de Propiedad Intelectual (SAPI), mantuvo actividades de formación en materia de propiedad intelectual para diversos entes del Estado, y reiteró su disposición de mantener el acompañamiento a la Denominación de Origen Cacao de Chuao otorgada en el año 2000.

\section{La propiedad industrial en Venezuela. Legislación internacional}

\section{Antecedentes de Venezuela en la Comunidad Andina (CAN)}

Es preciso señalar que la Comunidad Andina (CAN), es una organización subregional que posee personalidad jurídica de carácter internacional. Constituida actualmente por Bolivia, Colombia, Ecuador y Perú, así como, por el conjunto de órganos e instituciones que conforman el Sistema Andino de Integración (SAI). Dicho sistema permite que la Comunidad Andina se estructure y funcione muy similar a como se conforma y actúa un Estado, a partir de un conjunto de instituciones que tienen a su cargo funciones específicas.

Los antecedentes de la CAN se remontan a finales de la década de los 60's, específicamente, el 26 de mayo de 1969, año en que se firma el Acuerdo de Cartagena, también conocido como Pacto Andino o Grupo Andino. Años después, el 13 de febrero de 1973, Venezuela se adhirió al Acuerdo, hasta que el entonces Presidente de la República Bolivariana de Venezuela, Hugo Chávez, anunció su decisión de denunciar el Acuerdo de Cartagena, el 19 de abril de 2006. Es preciso acotar que el anuncio por parte de Venezuela, se realizó en un momento en el que el país ejercía la presidencia pro tempore del organismo andino, y se hizo efectivo, el 22 de abril del año 2011, debido a que una vez expresada la denuncia los países miembros debían preservar durante 5 años los beneficios del libre comercio.

La Decisión Andina sobre el Régimen Común de Propiedad Industrial, mejor conocida como la Decisión 486, del 14 de septiembre del año 2000, regula el otorgamiento de marcas y patentes, así como, la protección de los secretos industriales y las denominaciones de origen, entre otros. Esta Decisión sentó las bases para que los países miembros puedan contar con fuertes y modernos instrumentos legales para la defensa de los derechos de propiedad intelectual, cónsonos con las legislaciones internacionales en la materia. 
La aprobación de esta Decisión se presentó como una buena señal para los inversionistas de los países miembros y los extranjeros, en el sentido de la existencia de reglas del juego claras que incluyen procedimientos más ágiles y transparentes para los registros de marcas y el otorgamiento de patentes. Asimismo, es pertinente agregar que la Decisión 486 recoge los aspectos sobre el trato al nacional y a la nación más favorecida, términos y plazos, condiciones, idioma, derechos de prioridad, el desistimiento y abandono, trámites, tipos de licencias, concesión y nulidad de patentes, caducidad de los trámites y certificados, tipos de derechos y limitaciones, titularidad, medidas penales, actos de frontera, competencia desleal y secretos empresariales.

Pertenecer a la Comunidad Andina entre otros aspectos, otorgaba una de las mayores ventajas en cuanto a formar parte de uno de los organismos con una de las legislaciones más estructuradas en propiedad industrial, cuyos avances se han dado producto de las necesidades del entorno, haciendo hincapié en el valor que debe infringírsele a la generación y protección del conocimiento, más allá del mero hecho social, sino también desde una visión mucho más amplia, orientada a la generación de planes, alianzas, proyectos y asociaciones, cuyo fin último es el desarrollo de redes comerciales, industriales, empresariales públicas y privadas sólidas, que impulsen el desarrollo de los países.

Cuando Venezuela decide denunciar el Acuerdo de Cartagena, Tratado originario de la Comunidad Andina, no sólo se toma una medida desde la perspectiva política e ideológica, sino que convierte su resolución en una consecuencia que afectaría tanto a la propiedad industrial como a las relaciones y vinculaciones del país, con una de las alianzas que mayor beneficio comercial le prestaba a Venezuela. La causa de esta decisión sería los perjuicios inmediatos, que, según el ex Presidente Hugo Chávez, le causarían los Tratados Bilaterales de Libre Comercio (TLC), firmados por Colombia y Perú con Estados Unidos, a la alianza latinoamericana.

Según el análisis realizado por Carlos Malamud (2006), la salida de Venezuela de la CAN implicaría una participación más activa en otros bloques del continente, siendo el MERCOSUR el más cercano a los ideales políticos e ideológicos venezolanos. Esto ocasionó según el autor, una amenaza a la viabilidad de los dos grandes bloques de integración de América del Sur (CAN y MERCOSUR), abriendo una seria interrogante sobre el futuro de la Comunidad Sudamericana de Naciones (CSN), proyecto impulsado por Brasil, que se vio amenazado por dos acontecimientos posteriores, como la Cumbre de La Habana, en la cual el presidente Evo Morales decidió sumarse a la Alternativa Bolivariana para las Américas (ALBA), junto al decreto de nacionalización de los hidrocarburos bolivianos.

La importancia de las normas andinas para Venezuela debía analizarse según Sainz (2007), desde el orden internacional y el nacional. Están en juego un conjunto de compromisos internacionales que fueron suscritos por el país en los escenarios multilaterales, lo cual permitió la eficiente articulación de nuestro sistema comercial con el mundo, particularmente, a través de la Organización Mundial de Comercio (OMC), la Organización Internacional de la Propiedad Intelectual (OMPI) y otros organismos intergubernamentales que facilitan e intervienen en la actividad comercial mundial. 
Asimismo, es importante resaltar, que la primera norma subregional andina de Propiedad Industrial, fue la Decisión 311 del 1991, a esta le siguió la Decisión 313 de 1991, y luego la Decisión 314 de 1992, para culminar con la Decisión 486 del año 2000, aún vigente para los miembros del ente. De esta forma, las decisiones de la CAN prevalecían y se aplicaban en forma directa en Venezuela y dejaban sin efecto la antigua ley de Propiedad Industrial de 1956, incorporando a Venezuela a los más recientes institutos jurídicos sobre el tema, siguiendo el modelo legal adoptado en la Unión Europea y en la Organización Mundial de la Propiedad Intelectual la OMPI.

Al retirarse de la CAN, Venezuela dejó muchas interrogantes en cuanto a la aplicación de las normas en materia de propiedad industrial, recurriendo a la antigua ley de 1956, totalmente desfasada de los requerimientos, avances y necesidades de la sociedad moderna ya inmersa en los juegos de las relaciones internacionales del nuevo milenio. Con esta decisión, Venezuela fue parte de un gran retroceso en la materia, en la que se pierde entre otros aspectos, las denominaciones de origen, un proceso que posicionó a varios productos venezolanos como una referencia de calidad internacional. Cabe destacar que mientras Venezuela formó parte de la CAN se obtuvieron tres importantes denominaciones de origen, las cuales se indican a continuación:

a) Cacao de Chuao: fue la primera denominación de origen otorgada a una zona productora de cacao en Venezuela, reconocida por el Servicio Autónomo de la Propiedad Intelectual (SAPI), según Resolución No. 2006 de 14 de noviembre de 2000 y promovida por la Empresa Campesina Chuao, la Unidad Estadal del Ministerio del Poder Popular para la Agricultura y Tierras (UEMAT) de Aragua, la Fundación para el Desarrollo de la Ciencia y la Tecnología (FUNDACITE) Aragua y el Consejo de Desarrollo Económico, Tecnológico y de Exportación (CODET) de Aragua.

b) Cocuy Pecadero: segunda denominación de origen venezolana reconocida por el SAPI, según Resolución No. 0287 de 22 mayo de 2001. Concedida al principal producto de explotación del agave, planta que crece en la zona semiárida de Falcón (Pecaya, Municipio Sucre, al suroeste de la ciudad de Coro). Esta denominación contó con el impulso por parte de la Gobernación de Falcón, FUNDACITE Falcón, la Universidad Francisco de Miranda (Edo. Falcón) y la Asociación de Productores de Licor de Pecaya.

c) Ron de Venezuela: tercera denominación de origen venezolana, reconocida por el SAPI en la Resolución No. 798 de 15 agosto de 2003, y concedida a todos los rones de calidad que se producen en Venezuela, fue impulsada desde la Cámara de la Industria Venezolana de Especies Alcohólicas (CIVEA).

Hubo una cuarta denominación de origen que se tramitó y fue presentada ante el SAPI en el año 2005, pero lamentablemente dado que la denuncia se realiza en el año 2006, la misma no pudo ser otorgada. Esta fue la denominación de origen del Café Grano de Oro de Biscucuy, que era apoyada por la Alcaldía del Municipio Sucre del Estado Portuguesa.

\section{Marco jurídico internacional que rige a Venezuela hoy en materia de propiedad industrial}

Ahora bien, así como Venezuela posee un conjunto de políticas y leyes nacionales que debe aplicar, internacionalmente el país también debe cumplir con una legislación que según el 
Servicio Autónomo de Propiedad Intelectual (SAPI), rige al país en este sentido, y específicamente en lo que se refiere a la propiedad industrial a continuación se presenta este marco jurídico en la tabla 1:

Tabla 1. Marco jurídico internacional aplicable en materia de propiedad industrial en Venezuela

\begin{tabular}{ccc}
\hline Marco & Documento & Fecha \\
\hline \multirow{2}{*}{$\begin{array}{c}\text { Registro de } \\
\text { Marcas }\end{array}$} & Convenio que Establece la Organización Mundial de & Estocolmo \\
& Propiedad Intelectual (OMPI). & $14 / 07 / 1967$ \\
& Convenio de París para la Protección de la Propiedad & $20 / 03 / 1883$ \\
& Andustrial. & \\
& Acuerdo Sobre los Aspectos de los Derechos de Propiedad & $05 / 04 / 1994$ \\
Intelectual Relacionados con el Comercio (ADPIC). & Viena \\
Registro de & Acuerdo de Viena. & $12 / 06 / 1973$ \\
& Arreglo de Niza. & $15 / 06 / 1957$ \\
& Convenio que Establece la Organización Mundial de & Estocolmo \\
& Propiedad Intelectual. & $14 / 07 / 1967$ \\
& Convenio de París para la Protección de la Propiedad & $20 / 03 / 1883$ \\
& Acuerdo Sobre los Aspectos de los Derechos de Propiedad & $05 / 04 / 1994$ \\
\hline & Intelectual Relacionados con el Comercio (ADPIC). & Locarno \\
& Arreglo de Locarno. & $08 / 10 / 1968$ \\
& Arreglo de Estrasburgo. & Estrasburgo \\
\hline
\end{tabular}

Fuente: SAPI. Marco legal (s.f.). Recuperado el 25 de Enero, 2018, desde: http://www.sapi.gob.ve/.

Es oportuno destacar que en el Convenio de la Organización Mundial de la Propiedad Intelectual (OMPI), de 1967 se articulan todos los aspectos que organizan el sistema de propiedad intelectual a nivel mundial para la organización, estructura y funcionamiento, así como, el tratamiento a los países miembros, la sede, administración, capacidad jurídica y vinculación internacional del organismo.

El resto de los convenios, acuerdos y arreglos mencionados tratan sobre aspectos del registro de marcas y patentes a nivel internacional, incluyendo los sistemas de clasificación internacional de marcas dentro del Arreglo de Niza de 1957 y el Sistema de Clasificación Internacional de Patentes (CIP) de la OMPI.

Otra figura legal internacional a contemplar, es el Convenio $\mathrm{N}^{\circ} 169$ sobre Pueblos Indígenas y Tribales en Países Independientes de la Organización Internacional del Trabajo (OIT) de 1989. Este Convenio dispone en el artículo 31 que los pueblos indígenas tienen “...derecho a mantener, controlar, proteger y desarrollar su propiedad intelectual de dicho patrimonio cultural, sus conocimientos tradicionales y sus expresiones culturales tradicionales" (OIT, 1989: 96). Esta regulación internacional es concordante con lo dispuesto en la Constitución venezolana vigente de 1999 que preserva los conocimientos, tecnologías e innovaciones de los pueblos indígenas. 
Además, debe tomarse en cuenta que la Constitución Nacional establece en el artículo 153, la prevalencia de las normativas adoptadas en materia de integración que serán consideradas parte integrante del ordenamiento legal vigente y de aplicación directa y preferente a la legislación interna.

Asimismo, se requiere una participación más activa de los entes rectores venezolanos en las discusiones que sobre el tema se generen desde los diversos entes y alianzas regionales, e internacionales como el MERCOSUR, ALBA, UNASUR, la OMPI, los ADPIC o la OMC, como una herramienta de vital importancia al momento de generar insumos para adecuar de mejor manera y prontamente la ley de propiedad industrial del país.

Este proceso debe ser liderado por los entes nacionales que directa o indirectamente actúan sobre la materia, y aquellos receptores en segunda instancia de las decisiones tomadas desde el Estado, como son los institutos de investigación, universidades, empresas públicas y privadas, laboratorios, parques tecnológicos, innovadores, emprendedores, tecnólogos, que forman parte del SNCTI. Por tanto, es necesario avanzar más allá de un mero proceso de adecuación de la legislación, normas y leyes, sino también, de la generación de políticas públicas, instrumentos, incentivos actualizados que estimulen en el país y sus ciudadanos el conocimiento, valor, desarrollo, y perdurabilidad en el tiempo, de todos los elementos y razones existentes para hacer de la protección justa y equitativa del conocimiento nacional, un punto a favor de la cultura presente y futura de Venezuela.

\section{Conclusiones}

Como ha podido observarse precedentemente, la década de los 50`s fue crucial para la propiedad industrial en Venezuela, en ese período se dio inicio a su estructuración administrativa, se diseñaron las primeras directrices en materia de políticas públicas y la creación de la normativa que se erigiría en torno al tema. En principio esto fue señalado como un gran avance para el país, puesto que para la fecha estas acciones fueron renombradas en América Latina.

Las políticas públicas en cualquier sector, no deben basarse únicamente en leyes, normas y la creación de organismos, sino también deben propiciar la creación de programas, planes, proyectos, su administración, evaluación y seguimiento, y en este sentido, la propiedad industrial no escapa de ello. Hace falta generar espacios de interacción e intercambio que enriquezcan y fortalezcan un verdadero sistema de innovación nacional.

Es necesario concebir políticas públicas que refuercen el valor del conocimiento generado en el país y su protección, así como, impulsar la enseñanza y divulgación del área, y deben incorporarse las propuestas de otros sectores como el privado, el universitario, los centros e institutos de investigación y parques tecnológicos.

Por otro lado, debe advertirse que en el año 2012 se introdujo un nuevo mecanismo legal que incrementa la desarticulación entre el sector público y privado nacional. Ese dispositivo es la Ley Orgánica del Trabajo, los Trabajadores y las Trabajadoras (LOTTT), específicamente los artículos sobre la propiedad industrial, que dejan supeditada la protección del conocimiento a 
aspectos restringidos al tipo de financiamiento recibido, condicionando la libertad del mismo y el derecho de propiedad a quien realice la inversión para su desarrollo. Resulta propicio crear mecanismos certeros que incrementen la generación de productos y servicios cónsonos con las necesidades del venezolano, esto implica impulsar el desarrollo de la industria nacional, así como, la creación de una cultura exportadora.

En ese orden de ideas, las alianzas internacionales no necesariamente generan una respuesta, un cambio de ciertas circunstancias a lo interno de los países y sus instituciones, y por ende, aún menor podría ser su influencia en el comportamiento y respuesta final de los Estados frente a estos procesos. Kratochwill y Ruggie (1986), (citado en Keohane, 1988: 223) indican que:

Entender cómo piensa la gente acerca de las normas y reglas institucionales y el discurso en el que se comprometen, es tan importante para evaluar la significación de estas normas como para medir el comportamiento que cambia en respuesta a su invocación.

Los autores señalados muestran la persuasión que puede ejercer la naturaleza de los líderes sobre las instituciones y el viraje que pueden tomar los objetivos que inicialmente fueron una motivación para la integración.

Un punto de inflexión en este proceso es la suspensión aplicada a Venezuela por el MERCOSUR, el 2 de diciembre de 2016, emanada de la aplicación de la Convención de Viena sobre el Derecho de los Tratados del 23 de mayo de 1969, vigente desde el 27 de enero de 1980; como la activación del Protocolo de Ushuaia sobre Compromiso Democrático en el MERCOSUR, durante la reunión celebrada en Sao Paulo, el 5 de agosto de 2017, (MRECIC Argentina, $\left.\mathrm{N}^{\circ}: 316 / 17,2017\right)$, que llaman la atención sobre el estado de incumplimiento de Venezuela de las normas de MERCOSUR, y exhorta a la observancia de sus normas.

La propiedad industrial en Venezuela enfrenta peligros como la escasa vigilancia de los derechos de propiedad, la falta de monitoreo del cumplimiento y sanciones basadas en las leyes nacionales y los convenios y tratados internacionales, sumados a la piratería y el contrabando, que asfixian la creatividad y la innovación dejando a la intemperie a todo el conocimiento que se produzca en el país.

Esto motiva a que en la adecuación de las políticas y herramientas para el manejo de la propiedad intelectual y específicamente en la propiedad industrial, deben generarse cambios en cuanto al monitoreo que deben hacer los entes rectores en la materia sobre las acciones judiciales y el cumplimiento de la normativa para la observancia de estos derechos, con lo cual, se promoverá un uso y defensa de estos derechos como algo natural, y no como el producto de una actividad circunstancial o coyuntural.

Son muchas las leyes venezolanas que insertan en sus textos aspectos sobre la propiedad industrial, pero también, se observó la desconexión entre ellas, y entre éstas y la Ley de Propiedad Industrial de 1956, en lo que se refiere a los objetos de protección, y esto es producto de la antigüedad que experimenta el documento base frente a las leyes más recientes como la 
del trabajo, la ley de las comunas, sobre los pueblos y comunidades indígenas, la ley de aduanas, diversidad biológica, entre otras.

La estructura de las políticas públicas en propiedad industrial en Venezuela, requiere un cambio de enfoque que persiga en primer lugar, una formulación desde la inclusión y participación real de todos los sectores; en segundo lugar, llevar las políticas públicas en el tema a un nivel de competitividad internacional; en tercer lugar, que el ente rector en la materia, retome el papel para el cual fue creado y junto a otros actores del SNCTI, coordine las acciones que impulsen un Sistema Nacional de Innovación; en cuarto lugar, que se actualice la ley nacional y se analicen las normas y leyes conexas al tema, para que exista consonancia entre éstas y las instituciones involucradas en cada caso; y en quinto lugar, que se generen programas y planes para formar y promover el uso y beneficios de la propiedad industrial en todos los sectores del país.

\section{Bibliografía}

Asamblea Nacional Constituyente. (1999). Constitución de la República Bolivariana de Venezuela. Caracas.

Asamblea Nacional. (1999). Constitución de la República Bolivariana de Venezuela. (Gaceta Oficial de la República Bolivariana de Venezuela N 5.453-Extraordinaria), marzo 24, 2000.

Asamblea Nacional de la República Bolivariana de Venezuela. Ley de Diversidad Biológica. (Gaceta Oficial No 5.468). Mayo 24, 2000.

Asamblea Nacional. (2013). Plan de la Patria 2013-2019. (Gaceta Oficial de la República Bolivariana de Venezuela $N^{\circ}$ 6.118 Extraordinario), diciembre 4, 2013.

Comunidad Andina. (1991). Decisión 311 del Régimen Común sobre Propiedad Industrial. Caracas, Venezuela: Comisión del Acuerdo de Cartagena.

Comunidad Andina. (1992). Decisión 313 del Régimen Común sobre Propiedad Industrial. Quito, Ecuador: Comisión del Acuerdo de Cartagena.

Comunidad Andina. (1992). Decisión 314 Libertad de Acceso a las Cargas Transportadas por vía marítima y políticas para el desarrollo de la Marina Mercante del Grupo Andino. Quito, Ecuador: Comisión del Acuerdo de Cartagena.

Comunidad Andina. (1993). Decisión 345 del 21 de octubre de 1993. La Comisión del Acuerdo de Cartagena sobre el Régimen Común de Protección a los Derechos de los Obtentores de Variedades Vegetales. Bogotá, Colombia: Comisión del Acuerdo de Cartagena.

Comunidad Andina. (1996). Decisión 391 del 02 de Julio de 1996. Régimen Común sobre Acceso a los Recursos Genéticos. Caracas, Venezuela: Comisión del Acuerdo de Cartagena. 
Comunidad Andina. (2000). Decisión 486 del 14 de septiembre de 2000. Régimen Común sobre Propiedad Industrial. Lima, Perú: Comisión de la Comunidad Andina.

Comunidad Andina. (s/f). Recuperado el 18 de enero, 2018, desde: www.comunidadandina.org

Congreso de la República de Venezuela. (1955). Ley de Propiedad Industrial. (Gaceta Oficial N ${ }^{\circ}$ 25.227. Diciembre 10, 1956). Caracas.

Congreso de la República de Venezuela. Ley Orgánica de Procedimientos Administrativos. (Gaceta Oficial Extraordinaria $N^{\circ}$ 2818). Julio 01, 1981.

Congreso de la República de Venezuela. Ley de Diversidad Biológica. Gaceta Oficial Extraordinaria No 5.468). Mayo 24, 2000.

Consejo del Mercado Común. (1998). Protocolo de Ushuaia sobre Compromiso democrático en el MERCOSUR, la Republica de Bolivia y la Republica de Chile. Ushuaia.

Consejo del Mercado Común. (2005). Acuerdo Marco para la Adhesión de la República Bolivariana de Venezuela al MERCOSUR. Montevideo, diciembre 09, 2005.

Consejo Directivo del Observatorio Nacional de Ciencia Tecnología e Innovación (ONCTI). (2011). Reglamento del Programa de Estímulo a la Investigación (PEI). Reunión Extraordinaria $\mathrm{N}^{\circ} 04$ enero 12, 2011.Organización Mundial de la Propiedad Intelectual (OMPI). (s/f). Recuperado el 12 de Enero, 2018, desde: http://www.wipo.int/wipolex/es/results.jsp?countries=\&cat_id=12.

Keohane, R., y Nye, J. (1988). Poder e Interdependencia. Buenos Aires: Grupo Editor Latinoamericano.

Malamud, C. 2006. La salida de Venezuela de la Comunidad Andina de Naciones y sus repercusiones sobre la integración entre Naciones (1era. y 2da parte). Real Instituto Elcano de Estudios Internacionales y Estratégicos. Obtenido de: http://www.realinstitutoelcano.org/analisis/970.asp.

Ministerio del Poder Popular para Ciencia, Tecnología e Innovación. (2005). Plan Nacional de Ciencia, Tecnología e Innovación 2005-2030. Caracas: MPPCTI.

Ministerio del Poder Popular para Ciencia, Tecnología e Innovación. (2010). Ley Orgánica de Ciencia, Tecnología e Innovación. (Gaceta Oficial de la República Bolivariana de Venezuela, $\mathrm{N}^{\circ}$ 39.575), diciembre 8, 2010.

Ministerio del Poder Popular para Ciencia, Tecnología e Innovación. (2011). Reforma del Reglamento de la LOCTI. (Gaceta Oficial de la República Bolivariana de Venezuela $\mathrm{N}^{\circ}$ 39.795), noviembre 08, 2011. 
Ministerio del Poder Popular para Educación Universitaria, Ciencia y Tecnología. (2014). Documento de creación. (Gaceta $\mathrm{N}^{\circ} 40.489$ del 3 de septiembre de 2014, Decreto $\mathrm{N}^{\circ}$ 1.226). Caracas.

Ministerio del Poder Popular para el Comercio. (s/f). Recuperado el 08 de enero, 2018, desde: www.mincomercio.gob.ve.

Ministerio del Poder Popular para Educación Universitaria, Ciencia y Tecnología. (s/f). Recuperado el 08 de enero, 2018, desde: http://www.mppeuct.gob.ve/.

Ministerio del Poder Popular para el Trabajo. (2012). Ley Orgánica del Trabajo, los Trabajadores y las Trabajadoras. (Gaceta Oficial N 6.076), abril 30, 2012.

Ministerio del Poder Popular para las Comunas y los Movimientos Sociales. (2010). Ley Orgánica Del Sistema Económico Comunal. (Gaceta Oficial No 6.011, Extraordinario), diciembre $21,2010$.

Ministerio del Poder Popular para los Pueblos Indígenas. (2005). Ley Orgánica de los Pueblos y Comunidades Indígenas. (Gaceta Oficial N³8.344), diciembre 27, 2005.

Ministerio de Relaciones Exteriores y Culto (2017). Información para la Prensa $\mathrm{N}^{\circ}$ : 316/17. Buenos Aires. 2017, agosto 5. Recuperado el 06 de agosto, 2017, desde: http://www.cancilleria.gob.ar/decision-sobre-la- suspension-de-la-republica-bolivarianade-venezuela-en-el-mercosur-en-aplicacion.

Organización Mundial de la Propiedad Intelectual. (1957). Arreglo de Niza relativo a la Clasificación Internacional de Productos y Servicios para el Registro de las Marcas. Niza: OMPI.

Organización Mundial de la Propiedad Intelectual. (1967). Convenio de la Organización Mundial de la Propiedad Intelectual (OMPI). Estocolmo: OMPI.

Organización Internacional del Trabajo. (1989). Convenio de la Organización Internacional del Trabajo $N^{\circ} 169$ de Pueblos Indígenas y Tribales en Países Independientes. Ginebra: OIT.

Organización de las Naciones Unidas (1969). Convención de Viena sobre el Derecho de los Tratados. Recuperado el 12 de enero, 2017, desde: http://www.oas.org/xxxivga/spanish/reference_docs/convencion_viena.pdf.

Presidencia de la República Bolivariana de Venezuela. (2007). Proyecto Nacional Simón Bolívar Primer Plan Socialista (PPS) 2007-2013. Caracas: Presidencia de la República.

Presidencia de la República. Ley Orgánica de la Administración Pública. (Gaceta Oficial Extraordinaria $\mathrm{N}^{\circ}$ 6.147), noviembre 17, 2014.

Salazar, L. (2010). El Circuito Jurídico Económico de la Propiedad Intelectual. Caracas: CENDES. 
Sainz, J. (2007). La salida de Venezuela de la Comunidad Andina. Revista Politeia, Vol. $30 \mathrm{~N}^{\circ}$ $38 . \quad$ Caracas. Obtenido de: http://www2.scielo.org.ve/scielo.php?script=sci_arttext\&pid=S030397572007000100005 $\& \operatorname{lng}=\mathrm{es} \& \mathrm{nrm}=\mathrm{iso}$

Servicio Autónomo de Propiedad Intelectual (SAPI). (1997). Documento de creación. (Gaceta Oficial $N^{\circ} 36.192$ de fecha 24-04-97creado mediante el Decreto $\mathrm{N}^{\circ} 1.768$ de fecha 25 de marzo de 1997). Caracas.

Servicio Autónomo de Propiedad Intelectual. (2000). Resolución № 2006. Noviembre 14, 2000.

Servicio Autónomo de Propiedad Intelectual. (2001). Resolución Nº 0287. Mayo 22, 2001.

Servicio Autónomo de Propiedad Intelectual. (2003). Resolución N 798. Agosto 15, 2003.

Servicio Autónomo de Propiedad Intelectual. (s/f). Recuperado el 25 de enero, 2018, desde: http://www.sapi.gob.ve/.

Servicio Nacional Integrado de Administración Aduanera y Tributaria. Ley Orgánica de Aduanas. (Gaceta Oficial N 5.353 Extraordinario), junio 17, 1999.

Zurbriggen, C., y González, M. (2010). Políticas de Ciencia, Tecnología e Innovación en los países del MERCOSUR. CEFIR Integración Regional. № 006: Montevideo. 13-48. 